\title{
q-Hook formula of Gansner type for a generalized Young diagram
}

\author{
Kento Nakada
}

Wakkanai Hokusei Gakuen University, Faculty of Integrated Media.nakada@wakhok.ac.jp

\begin{abstract}
The purpose of this paper is to present the $q$-hook formula of Gansner type for a generalized Young diagram in the sense of D. Peterson and R. A. Proctor. This gives a far-reaching generalization of a hook length formula due to J. S. Frame, G. de B. Robinson, and R. M. Thrall. Furthurmore, we give a generalization of P. MacMahon's identity as an application of the $q$-hook formula.

Résumé. Le but de ce papier est présenter la q-hook formule de type Gansner pour un Young diagramme généralisé dans le sens de D. Peterson et R. A. Proctor. Cela donne une généralisation de grande envergure d'une hook length formule dû à J. S. Frame, G. de B. Robinson, et R. M. Thrall. Furthurmore, nous donnons une généralisation de l'identité de P. MacMahon comme une application de la q-hook formule.
\end{abstract}

Keywords: Generalized Young diagrams, Trace generating functions, $q$-hook formula, Kac-Moody Lie algebra, P. MacMahon's identity

\section{Introduction}

In [3], E. R. Gansner proved a multivariable q-hook formula for a Young diagram $Y$ :

$$
\sum_{\sigma: \text { reverse plane partition over } Y} \mathbf{q}^{\sigma}=\prod_{v \in Y} \frac{1}{1-\mathbf{q}^{\mathrm{H}(v)}},
$$

where $\mathrm{H}(v)$ denotes the hook of a cell $v \in Y$ (see section 2 and 3 for a precise definition). The identity [1.1] is a multi- $q$-refinement of the famous hook length formula [2]

$$
\# \operatorname{STab}(Y)=\frac{(\# Y) !}{\prod_{v \in Y} \# \mathrm{H}(v)},
$$

due to J. S. Frame, G. de B. Robinson, and R. M. Thrall.

The purpose of this paper is to present a generalization of (1.1) for a generalized Young diagram in the sense of D. Peterson and R. A. Proctor. Our (multivariable) $q$-hook formula is:

$$
\sum_{\sigma:\left(\mathrm{D}(\lambda)^{\vee} ; \leq\right) \text {-partition }} \mathbf{q}^{\sigma}=\prod_{\beta^{\vee} \in \mathrm{D}(\lambda)^{\vee}} \frac{1}{1-\mathbf{q}^{\mathrm{H}_{\lambda}\left(\beta^{\vee}\right)}},
$$

1365-8050 (c) 2009 Discrete Mathematics and Theoretical Computer Science (DMTCS), Nancy, France 
where $\lambda$ is a finite pre-dominant integral weight for a Kac-Moody Lie algebra and $\mathrm{D}(\lambda)^{\vee}$ is a certain set of real coroots (see section 6 for more details). We note that the identity 1.3 is equivalent with unpublished result by D. Peterson and R. A. Proctor [11].

Similarly, the identity $(1.3)$ implies a hook length formula:

$$
\mathcal{L}\left(\mathrm{D}(\lambda)^{\vee}\right)=\prod_{\beta^{\vee} \in \mathrm{D}(\lambda)^{\vee}} \frac{\left(\# \mathrm{D}(\lambda)^{\vee}\right) !}{\prod_{\beta^{\vee} \in \mathrm{D}(\lambda)^{\vee}} \# \mathrm{H}_{\lambda}\left(\beta^{\vee}\right)},
$$

where $\mathcal{L}\left(\mathrm{D}(\lambda)^{\vee}\right)$ denotes the set of linear extensions (or reverse standard tableaux) of the poset $\left(\mathrm{D}(\lambda)^{\vee} ; \leq\right.$ ). We note that the identity (1.4) is also equivalent with an unpublished result (see [1]) by D. Peterson, namely that:

$$
\# \operatorname{Red}(w)=\frac{\ell(w) !}{\prod_{\beta \in \Phi(w)} \operatorname{ht}(\beta)},
$$

a hook formula for the number of reduced decompositions of a minuscule elements $w$ of the Kac-Moody Weyl group, where $\operatorname{Red}(w)$ denotes the set of reduced decompositions of $w, \ell(w)$ denotes the length of $w, \Phi(w)$ denotes the inversion set of $w$ :

$$
\Phi(w)=\left\{\beta \in \Phi_{+} \mid w^{-1}(\beta)<0\right\},
$$

and ht $(\beta)$ denotes the height of $\beta$.

\section{$2(P ; \leq)$-Partitions and $(c ; I)$-Trace generating functions}

Let $P=(P ; \leq)$ be a finite partially ordered set.

Definition 2.1 A map $\sigma: P \longrightarrow \mathbb{N}=\{0,1,2, \cdots\}$ is said to be a $(P ; \leq)$-partition if:

For each $u, v \in P$ such that $u \leq v$, we have $\sigma(u) \geq \sigma(v)$.

The set of $(P ; \leq)$-partitions is denoted by $\mathrm{A}(P ; \leq)$.

Let $I$ be a finite color-set (just a set). Let $c: P \longrightarrow I$ be a coloring (just a map). Let $q_{i}$ be an indeterminate indexed by a color $i \in I$. For each $\sigma \in \mathrm{A}(P ; \leq)$, we define a monomial $\mathbf{q}^{\sigma}$ by:

$$
\mathbf{q}^{\sigma}:=\prod_{v \in P} q_{c(v)}^{\sigma(v)} .
$$

We define a formal power series $T(P ; \leq)$ by:

$$
T(P ; \leq):=\sum_{\sigma \in \mathrm{A}(P ; \leq)} \mathbf{q}^{\sigma} .
$$

We call $T(P ; \leq)$ the $(c ; I)$-trace generating function of $(P ; \leq)$.

Definition 2.2 Put $d:=\# P$. A bijection $L:\{1, \cdots, d\} \longrightarrow P$ is said to be a linear extension (or reverse standard tableau) of $(P ; \leq)$ if:

$$
L(k) \leq L(l) \text { implies } k \leq l, \quad k, l \in\{1, \cdots, d\} .
$$

The set of linear extensions of $(P ; \leq)$ is denoted by $\mathcal{L}(P ; \leq)$. 
Let $q$ be another indeterminate. When we take the specialization $q_{i} \longmapsto q(i \in I)$, we denote $T(P ; \leq)$ by $U(P ; \leq)$.

Proposition 2.3 (R. P. Stanley [12]) We have:

$$
U(P ; \leq)=\frac{W(P ; q)}{\prod_{k=1}^{d}\left(1-q^{k}\right)},
$$

for some $W(P ; q) \in \mathbb{Z}[q]$. Furthermore, we have $W(P ; 1)=\# \mathcal{L}(P ; \leq)$.

Remark 2.4 In section 7, we consider a certain infinite partially ordered set with a certain infinte colorset I. In such a situation, we define a notion of $(P ; \leq)$-partitions as follows:

We define a lattice $Q$ by:

$$
Q=\bigoplus_{i \in I} \mathbb{Z} \alpha_{i}
$$

where $\left\{\alpha_{i} \mid i \in I\right\}$ is a formal basis. A map $\sigma: P \longrightarrow \mathbb{N}$ is said to be a $(P ; \leq)$-partition if:

1. For each $u, v \in P$ such that $u \leq v$, we have $\sigma(u) \geq \sigma(v)$.

2. There exists at most finitely many $v \in P$ such that $\sigma(v) \geq 1$.

The set of $(P ; \leq)$-partitions is denoted by $\mathrm{A}(P ; \leq)$. A (possibly infinite) partially ordered set $(P ; \leq)$ is said to be a $(c ; I)$-compatible poset if:

For each $\phi \in Q$, there exists at most finitely many $\sigma \in \mathrm{A}(P ; \leq)$ such that $\sum_{v \in P} \sigma(v) \alpha_{c(v)}=\phi$.

\section{Case of Young diagrams}

When we draw a Young diagram, we use nodes instead of cells like FIGURE3.1 (left) below:
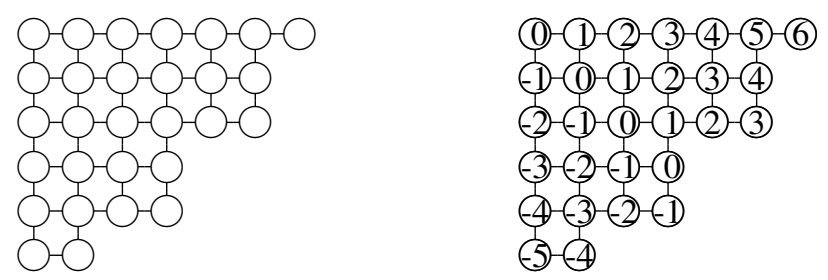

Fig. 3.1: a Young diagram and its coloring

Definition 3.1 We equip the set $\mathbb{Y}:=\mathbb{N} \times \mathbb{N}$ with the partial order:

$$
(i, j) \leq\left(i^{\prime}, j^{\prime}\right) \Longleftrightarrow i \geq i^{\prime} \text { and } j \geq j^{\prime} .
$$

A finite order filter $Y$ of $\mathbb{Y}$ is called a Young diagram. 
Definition 3.2 Put $I:=\mathbb{Z}$ as a color-set. For each node $v=(i, j) \in Y$, we attach the color $c(v)$ by:

$$
c(v):=j-i \in I
$$

see FIGURE 3.1 (right). The color $c(v)$ is known as the content of $v$.

In the case of above example, we can take

$$
I=\{-6,-5,-4,-3,-2,-1,0,1,2,3,4,5,6\},
$$

as finite color-set.

Definition 3.3 Let $Y$ be a Young diagram. Let $v=(i, j) \in Y$. We define the subset $\mathrm{H}(v)$ of $Y$ by:

$$
\begin{gathered}
\operatorname{Arm}(v):=\left\{\left(i^{\prime}, j^{\prime}\right) \in Y \mid i=i^{\prime} \text { and } j<j^{\prime}\right\} . \\
\operatorname{Leg}(v):=\left\{\left(i^{\prime}, j^{\prime}\right) \in Y \mid i<i^{\prime} \text { and } j=j^{\prime}\right\} . \\
\mathrm{H}(v):=\{v\} \sqcup \operatorname{Arm}(v) \sqcup \operatorname{Leg}(v) .
\end{gathered}
$$

The set $\mathrm{H}(v)$ is called the hook of $v \in Y$ (see FIGURE 3.2).
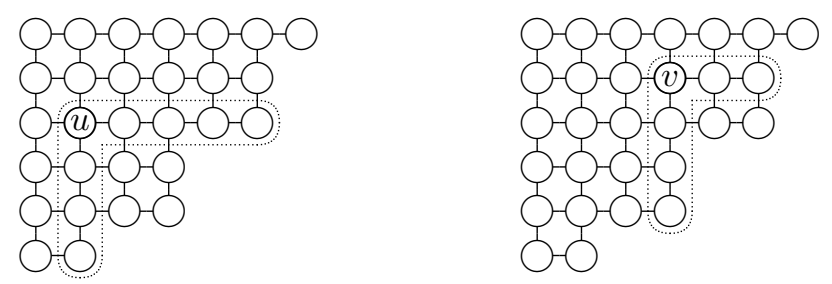

Fig. 3.2: Hooks of $u$ and $v$

Then we have the following theorem:

Theorem 3.4 (E. R. Gansner [3]) Let $Y=(Y ; \leq)$ be a Young diagram. Then we have:

$$
T(Y ; \leq)=\prod_{v \in Y} \frac{1}{1-\mathbf{q}^{\mathrm{H}(v)}},
$$

where $\mathbf{q}^{\mathrm{H}(v)}=\prod_{u \in \mathrm{H}(v)} q_{c(u)}$.

Remark $3.5 A(Y ; \leq)$-partition is called a reverse plane partition over $Y$.

\section{Case of shifted Young diagrams}

Definition 4.1 We equip the $\mathbb{S}:=\{(i, j) \in \mathbb{N} \times \mathbb{N} \mid i \leq j\}$ with the partial order:

$$
(i, j) \leq\left(i^{\prime}, j^{\prime}\right) \Longleftrightarrow i \geq i^{\prime} \text { and } j \geq j^{\prime}
$$

A finite order filter $S$ of $\mathbb{S}$ is called a shifted Young diagram. 


\subsection{Case of Shifted Young Diagrams with standard hooks}

Definition 4.2 Put $I:=\{\infty\} \cup \mathbb{N}$ as the color-set. For each node $v=(i, j) \in S$, we define the color $c(v)$ by:

$$
c(v)= \begin{cases}0 & \text { if } i=j \text { and } i \text { is even } \\ \infty & \text { if } i=j \text { and } i \text { is odd } \\ j-i & \text { if } i<j .\end{cases}
$$

see FIGURE 4.1]

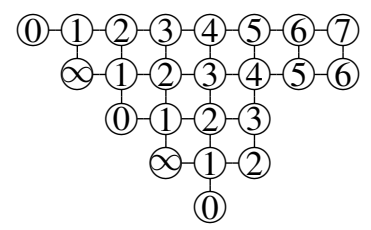

Fig. 4.1: Colors of the nodes of a shifted Young diagram

In the case of the above example, we can take $I=\{\infty, 0,1,2,3,4,5,6,7\}$ as finite color-set.

Definition 4.3 Let $S$ be a shifted Young diagram. Let $v=(i, j) \in S$. We define the subset $\mathrm{H}_{\mathrm{D}}(v)$ of $S$ by:

$$
\begin{aligned}
\operatorname{Arm}_{\mathrm{D}}(v) & :=\left\{\left(i^{\prime}, j^{\prime}\right) \in S \mid i=i^{\prime} \text { and } j<j^{\prime}\right\} . \\
\operatorname{Leg}_{\mathrm{D}}(v) & :=\left\{\left(i^{\prime}, j^{\prime}\right) \in S \mid i<i^{\prime} \text { and } j=j^{\prime}\right\} . \\
\operatorname{Tail}_{\mathrm{D}}(v) & :=\left\{\left(i^{\prime}, j^{\prime}\right) \in S \mid j+1=i^{\prime} \text { and } j<j^{\prime}\right\} . \\
\mathrm{H}_{\mathrm{D}}(v) & :=\{v\} \sqcup \operatorname{Arm}_{\mathrm{D}}(v) \sqcup \operatorname{Leg}_{\mathrm{D}}(v) \sqcup \operatorname{Tail}_{\mathrm{D}}(v) .
\end{aligned}
$$

The set $\mathrm{H}_{\mathrm{D}}(v)$ is called the hook (of type $D$ ) of $v \in S$ (see FIGURE 4.2).

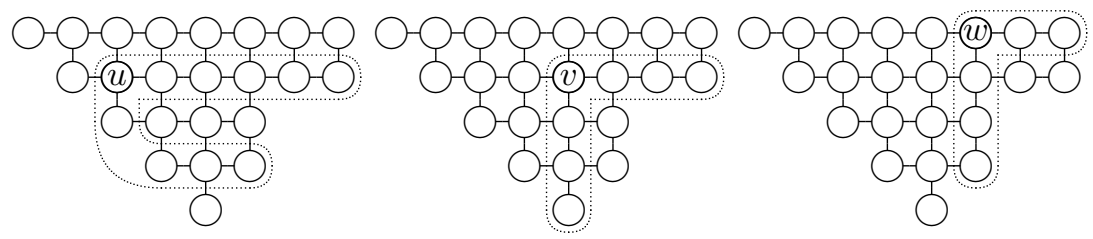

Fig. 4.2: Hooks of $u, v$, and $w$.

Then we have the following theorem:

Theorem 4.4 Let $S=(S ; \leq)$ be a shifted Young diagram with the coloring defined above. Then we have:

$$
T(S ; \leq)=\prod_{v \in S} \frac{1}{1-\mathbf{q}^{\mathrm{H}_{\mathrm{D}}(v)}} .
$$

Identifying $q_{\infty}$ with $q_{0}$ in Theorem 4.4, we get the following theorem obtained by Gansner: 
Theorem 4.5 (E. R. Gansner [3]) Let $S=(S ; \leq)$ be a shifted Young diagram. Then we have:

$$
\left.T(S ; \leq)\right|_{q_{\infty}=q_{0}}=\left.\prod_{v \in S} \frac{1}{1-\mathbf{q}^{\mathrm{H}_{\mathrm{D}}(v)}}\right|_{q_{\infty}=q_{0}} .
$$

Remark 4.6 The proof of Theorem 4.5 by Gansner is by Hillman-Grassl algorithm [4] based on hooks of type $D$.

\subsection{Case of Shifted Young Diagrams with non-standard hooks}

Definition 4.7 Put $I:=\mathbb{N}$ as the color-set. For each node $v=(i, j) \in S$, we define the color $c(v)$ by:

$$
c(v)=j-i,
$$

see FIGURE 4.3

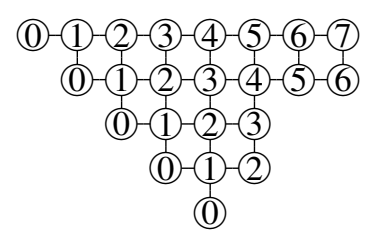

Fig. 4.3: Colors of the nodes of a shifted Young diagram

In the case of the above example, we can take $I=\{0,1,2,3,4,5,6,7\}$ as a finite color-set.

Definition 4.8 Let $S$ be a shifted Young diagram. Let $v=(i, j) \in S$. We define a subset $\mathrm{H}_{\mathrm{B}}(v)$ of $S$ by:

$$
\begin{aligned}
& \operatorname{Arm}_{\mathrm{B}}(v):=\left\{\left(i^{\prime}, j^{\prime}\right) \in S \mid i=i^{\prime} \text { and } j<j^{\prime}\right\} . \\
& \operatorname{Leg}_{\mathrm{B}}(v):=\left\{\left(i^{\prime}, j^{\prime}\right) \in S \mid i<i^{\prime} \text { and } j=j^{\prime}\right\} . \\
& \operatorname{Tail}_{\mathrm{B}}(v):= \begin{cases}\{(i, i)\} \sqcup\left\{\left(i^{\prime}, j^{\prime}\right) \in S \mid j=i^{\prime} \text { and } j<j^{\prime}\right\} & \text { if } i<j \text { and }(j, j) \in S, \\
\varnothing & \text { otherwise. }\end{cases} \\
& \qquad \mathrm{H}_{\mathrm{B}}(v):=\{v\} \sqcup \operatorname{Arm}_{\mathrm{B}}(v) \sqcup \operatorname{Leg}_{\mathrm{B}}(v) \sqcup \operatorname{Tail}_{\mathrm{B}}(v) .
\end{aligned}
$$

The set $\mathrm{H}_{\mathrm{B}}(v)$ is called a hook (of type $B$ ) of $v \in S$ (see FIGURE 4.4).
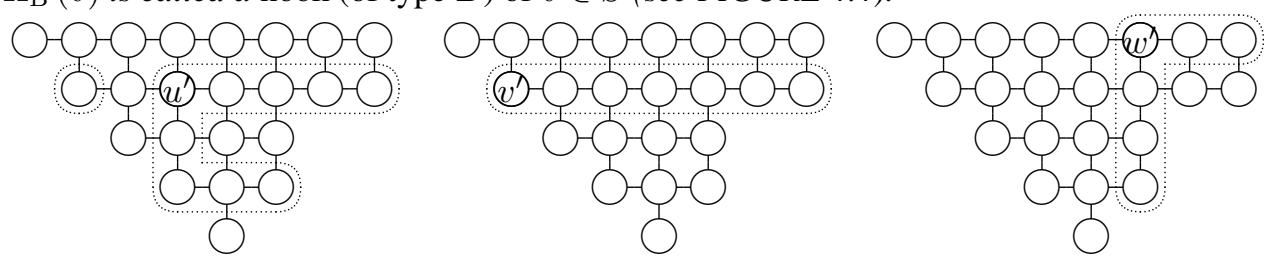

Fig. 4.4: Hooks of $u^{\prime}, v^{\prime}$, and $w^{\prime}$.

Remark 4.9 The nodes $u^{\prime}, v^{\prime}, w^{\prime}$ in FIGURE 4.4 corresponds to $u, v, w$ in FIGURE 4.2 in the sense of Remark 4.11 
Then we have the following theorem:

Theorem 4.10 Let $S=(S ; \leq)$ be a shifted Young diagram with the coloring defined above. Then we have:

$$
T(S ; \leq)=\prod_{v \in S} \frac{1}{1-\mathbf{q}^{\mathrm{H}_{\mathrm{B}}(v)}} .
$$

Remark 4.11 Let $S=(S ; \leq)$ be a shifted Young diagram. Then, there exists a bijection $S \ni v \mapsto v^{\prime} \in S$ such that $\mathbf{q}^{\mathrm{H}_{\mathrm{B}}\left(v^{\prime}\right)}=\left.\mathbf{q}^{\mathrm{H}_{\mathrm{D}}(v)}\right|_{q_{\infty}=q_{0}}(v \in S)$. Hence, Theorem 4.10 is same as Theorem 4.5 except for "shapes" of hooks.

\section{Case of the bat}

Definition 5.1 Let Bat $=($ Bat; $\leq)$ be the poset depicted in FIGURE 5.1 left $)$ below. The poset Bat is called the bat.

Definition 5.2 Put $I:=\{1,2,3,4,5,6,7\}$ as the color-set. The color of each vertex is written in the vertex in FIGURE 5.1 right) below.

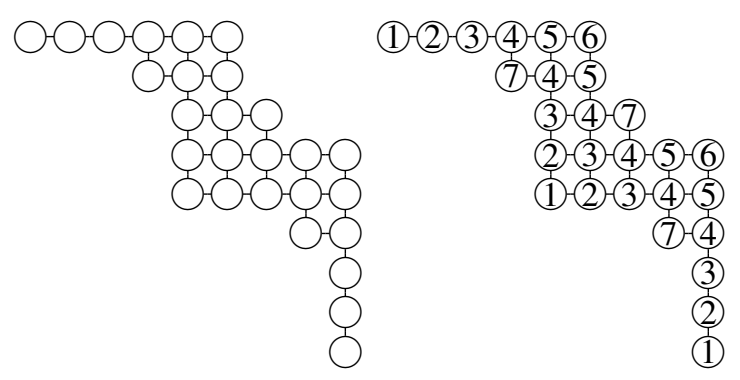

Fig. 5.1: The Bat, and the colors of the nodes of the Bat

Definition 5.3 The hook $\mathrm{H}_{\mathrm{Bat}}(v)$ of $v \in$ Bat is defined as in FIGURE 5.2 below.

Then we have the following theorem:

Theorem 5.4 The bat $\mathrm{Bat}=(\mathrm{Bat} ; \leq)$ with the colors defined above satisfies:

$$
T(\mathrm{Bat} ; \leq)=\prod_{v \in \mathrm{Bat}} \frac{1}{1-\mathbf{q}^{\mathrm{H} \text { Bat }}(v)} .
$$

\section{$6 q$-Hook formula of Gansner type for a generalized Young dia- gram}

In this section, we fix a Kac-Moody Lie algebra $\mathfrak{g}$ with a simple root system $\Pi=\left\{\alpha_{i} \mid \in I\right\}$. For all undefined terminology in this section, we refer the reader to [5] [7]. 


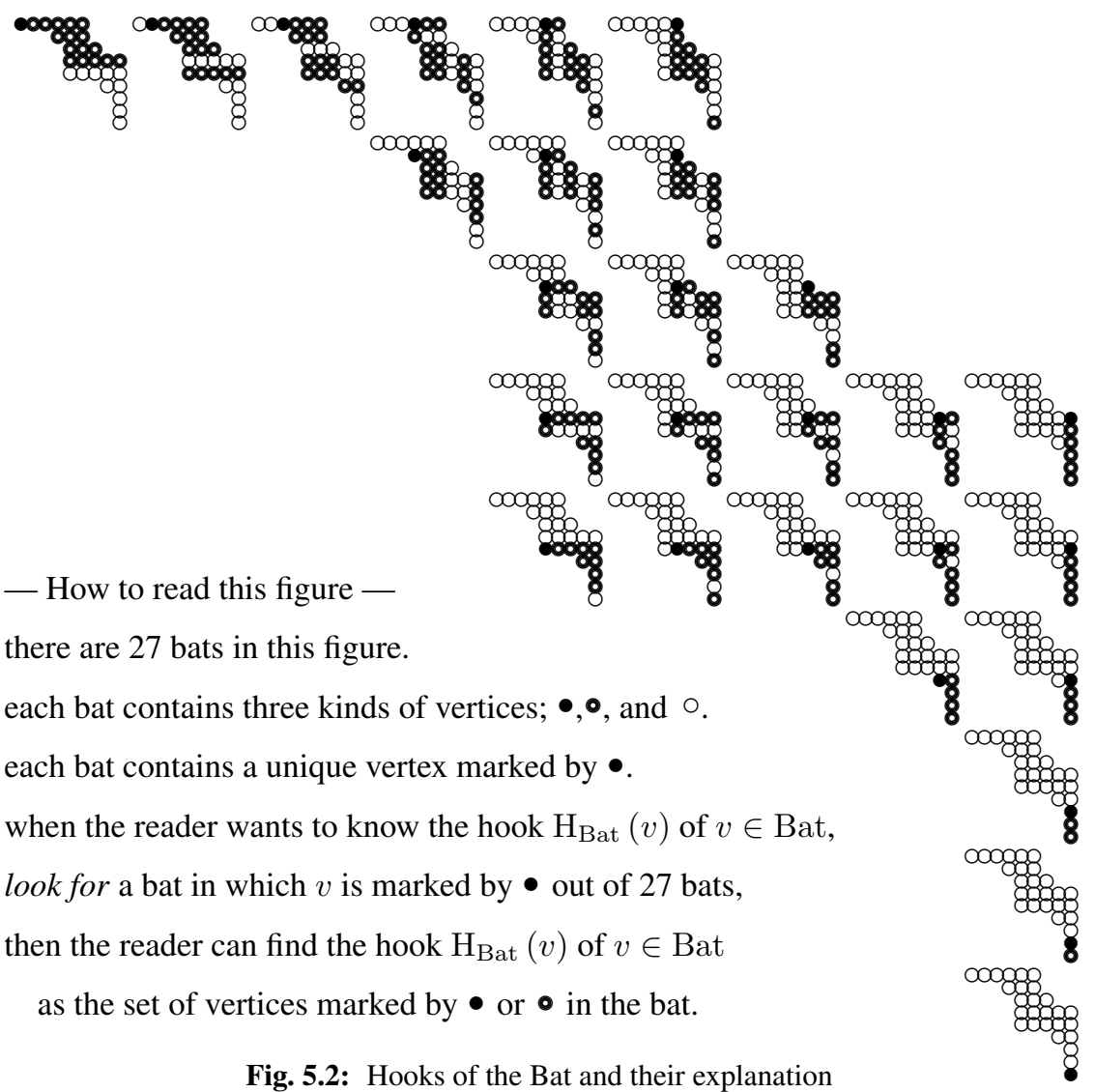

Definition 6.1 An integral weight $\lambda$ is said to be pre-dominant if:

$$
\left\langle\lambda, \beta^{\vee}\right\rangle \geq-1 \quad \text { for each } \beta^{\vee} \in \Phi_{+}^{\vee},
$$

where $\Phi_{+}^{\vee}$ denotes the set of positive real coroots. The set of pre-dominant integral weights is denoted by $P_{\geq-1}$. For $\lambda \in P_{\geq-1}$, we define the set $\mathrm{D}(\lambda)^{\vee}$ by:

$$
\mathrm{D}(\lambda)^{\vee}:=\left\{\beta^{\vee} \in \Phi_{+}^{\vee} \mid\left\langle\lambda, \beta^{\vee}\right\rangle=-1\right\} .
$$

The set $\mathrm{D}(\lambda)^{\vee}$ is called the shape of $\lambda$. If $\# \mathrm{D}(\lambda)^{\vee}<\infty$, then $\lambda$ is called finite. We regard the set $\mathrm{D}(\lambda)^{\vee}$ as a poset with coroot order $\leq$.

\subsection{Colors}

We regard the index set $I$ of simple roots as a color-set.

Definition 6.2 Let $\lambda \in P_{\geq-1}$ be finite. Put $d:=\# \mathrm{D}(\lambda)^{\vee}$. A sequence $\left(\alpha_{i_{1}}, \cdots, \alpha_{i_{d}}\right)$ of simple roots is said to be a maximal $\lambda$-path if:

$$
\left\langle s_{i_{k-1}} \cdots s_{i_{1}}(\lambda), \alpha_{i_{k}}^{\vee}\right\rangle=-1, \quad k=1, \cdots, d .
$$


The set of maximal $\lambda$-paths is denoted by $\operatorname{MPath}(\lambda)$.

Proposition 6.3 (see [8]) Let $\lambda \in P_{\geq-1}$ be finite. Then

1. We have $\operatorname{MPath}(\lambda) \neq \varnothing$.

2. Let $\beta^{\vee} \in \mathrm{D}(\lambda)^{\vee}$. Let $\left(\alpha_{i_{1}}, \cdots, \alpha_{i_{d}}\right) \in \operatorname{MPath}(\lambda)$. Then there exists a unique $k \in\{1, \cdots, d\}$ such that

$$
s_{i_{1}} \cdots s_{i_{k-1}}\left(\alpha_{i_{k}}^{\vee}\right)=\beta^{\vee} .
$$

3. In Part (2), the index $i_{k} \in I$ only depends on $\beta^{\vee}$. (Namely, $i_{k} \in I$ is independent from the choice of maxmal $\lambda$-path.)

Definition 6.4 Let $\lambda \in P_{\geq-1}$ and $\beta^{\vee} \in \mathrm{D}(\lambda)^{\vee}$. Then we have a unique index $i \in I$ corresponding to $\beta^{\vee}$ in the sense of Proposition 6.3. We denote such $i \in I$ by $c_{\lambda}\left(\beta^{\vee}\right)$. We call $c_{\lambda}\left(\beta^{\vee}\right)$ the color of $\beta^{\vee}$.

\subsection{Hooks}

Definition 6.5 Let $\lambda \in P_{\geq-1}$ and $\beta^{\vee} \in \mathrm{D}(\lambda)^{\vee}$. We define the set $\mathrm{H}_{\lambda}(\beta)^{\vee}$ by:

$$
\mathrm{H}_{\lambda}\left(\beta^{\vee}\right):=\mathrm{D}(\lambda)^{\vee} \cap \Phi\left(s_{\beta}\right)^{\vee} .
$$

where $\Phi\left(s_{\beta}\right)^{\vee}$ denotes the inversion set of the reflection corresponding to $\beta$ :

$$
\Phi\left(s_{\beta}\right)^{\vee}=\left\{\gamma^{\vee} \in \Phi_{+}^{\vee} \mid s_{\beta}\left(\gamma^{\vee}\right)<0\right\} .
$$

Proposition 6.6 (see [8]) Let $\lambda \in P_{\geq-1}$ be finite and $\beta^{\vee} \in \mathrm{D}(\lambda)^{\vee}$. Then we have:

1. $\sum_{\gamma^{\vee} \in \mathrm{H}_{\lambda}\left(\beta^{\vee}\right)} \alpha_{c_{\lambda}\left(\gamma^{\vee}\right)}=\beta$.

2. $\# \mathrm{H}_{\lambda}\left(\beta^{\vee}\right)=\operatorname{ht}(\beta)$.

\subsection{Main Theorem and Corollaries}

Theorem 6.7 (see [ [8]) Let $\lambda \in P_{\geq-1}$ be finite. Then we have:

$$
T\left(\mathrm{D}(\lambda)^{\vee} ; \leq\right)=\prod_{\beta^{\vee} \mathrm{D}(\lambda)^{\vee}} \frac{1}{1-\mathbf{q}^{\mathrm{H}_{\lambda}\left(\beta^{\vee}\right)}}=\prod_{\beta^{\vee} \mathrm{D}(\lambda)^{\vee}} \frac{1}{1-\mathbf{q}^{\beta}} .
$$

Taking the specialization $q_{i} \longmapsto q$, we get:

Corollary 6.8 Let $\lambda \in P_{\geq-1}$ be finite. Then we have:

$$
U\left(\mathrm{D}(\lambda)^{\vee} ; \leq\right)=\prod_{\beta^{\vee} \mathrm{D}(\lambda)^{\vee}} \frac{1}{1-q^{\# \mathrm{H}_{\lambda}\left(\beta^{\vee}\right)}}=\prod_{\beta^{\vee} \in \mathrm{D}(\lambda)^{\vee}} \frac{1}{1-q^{\mathrm{ht}(\beta)}} .
$$

Remark 6.9 A statement equivalent with Corollary 6.8 is also given in [11].

Hence, by Proposition 2.3 we get:

Corollary 6.10 Let $\lambda \in P_{\geq-1}$ be finite. Put $d:=\# \mathrm{D}(\lambda)^{\vee}$. Then we have: 

1. $W(P ; q)=\frac{\prod_{k=1}^{d}\left(1-q^{k}\right)}{\prod_{\beta^{\vee} \in \mathrm{D}(\lambda)^{\vee}}\left(1-q^{\# \mathrm{H}_{\lambda}\left(\beta^{\vee}\right)}\right)}=\frac{\prod_{k=1}^{d}\left(1-q^{k}\right)}{\prod_{\beta^{\vee} \in \mathrm{D}(\lambda)^{\vee}}\left(1-q^{\mathrm{ht}(\beta)}\right)}$.
2. $\# \mathcal{L}\left(\mathrm{D}(\lambda)^{\vee} ; \leq\right)=\frac{d !}{\prod_{\beta^{\vee} \in \mathrm{D}(\lambda)^{\vee}} \# \mathrm{H}_{\lambda}\left(\beta^{\vee}\right)}=\frac{d !}{\prod_{\beta^{\vee} \in \mathrm{D}(\lambda)^{\vee}} \mathrm{ht}(\beta)}$.

Remark 6.11 All shapes explaind in section 3,4, and 5 are realized as shapes of some $\lambda \in P_{\geq-1}$ over some root systems of finite types. Furthermore, colors and hooks defined in section 3,4, and 5 are compatible with those defined in this section.

* A Young diagram is realized over a root system of type A.

* A shifted Young diagram with hooks of type D is realized over a root system of type D.

* A shifted Young diagram with hooks of type B is realized over a root system of type B.

* The bat (or an order filter of the bat) is realized over a root system of type $E_{7}$.

There are 17 classes of generalized Young diagrams (15 of 17 are simply-laced). We note that many of them are realized over root systems of indefinite types (see [10] [13]).

Remark 6.12 Corollary 6.10 (2) gives a proof of Peterson's hook formula. Another proof of Peterson's hook formula is given in [9].

\section{An application to infinite rank case}

Although Theorem 6.7 holds for a finite pre-dominant integral weight, there exist several cases where Theorem 6.7 holds for an infinite pre-dominant integral weight.

Let $A_{\infty}$ denote the Dynkin diagram depicted below:

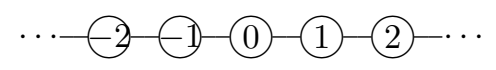

Here, an integer in a vertex is the index of the vertex. Let $\omega_{i}$ denote the fundamental weight corresponding to an index $i \in \mathbb{Z}$. Let $\lambda=\sum_{i \in \mathbb{Z}} c_{i} \omega_{i}$ be an integral weight satisfying the following properties:

1. For each $i \in \mathbb{Z}$, we have $c_{i} \in\{1,0,-1\}$.

2. Let $i, j \in \mathbb{Z}$ satisfy $i<j$ and $c_{i}=c_{j}= \pm 1$. Then we have $c_{n}=\mp 1$ for some $i<n<j$.

3. There exists at least one and at most finitely many $i \in \mathbb{Z}$ such that $c_{i} \neq 0$.

4. Let $i \in \mathbb{Z}$ be the minimum (or maximum) integer such that $c_{i} \neq 0$. Then we have $c_{i}=-1$.

5. (normalization) We have $\sum_{i \in \mathbb{Z}} c_{i} \cdot i=0$.

Then $\lambda$ is an infinite pre-dominant integral weight. The poset $\left(D(\lambda)^{\vee} ; \leq\right)$ is $\left(c_{\lambda} ; \mathbb{Z}\right)$-compatible. Furthermore, the statement of Theorem 6.7 holds for this $\lambda$ :

$$
T\left(\mathrm{D}(\lambda)^{\vee} ; \leq\right)=\prod_{\beta^{\vee} \in \mathrm{D}(\lambda)^{\vee}} \frac{1}{1-\mathbf{q}^{\mathrm{H}_{\lambda}\left(\beta^{\vee}\right)}}=\prod_{\beta^{\vee} \in \mathrm{D}(\lambda)^{\vee}} \frac{1}{1-\mathbf{q}^{\beta}} .
$$


Taking the specialization $q_{i} \longmapsto q(i \in \mathbb{Z})$ in 7.1 , we get:

$$
U\left(\mathrm{D}(\lambda)^{\vee} ; \leq\right)=\prod_{\beta^{\vee} \in \mathrm{D}(\lambda)^{\vee}} \frac{1}{1-q^{\# \mathrm{H}_{\lambda}\left(\beta^{\vee}\right)}}=\prod_{\beta^{\vee} \in \mathrm{D}(\lambda)^{\vee}} \frac{1}{1-q^{\mathrm{ht}(\beta)}} .
$$

In particular, we let $\lambda=-\omega_{0}$, which obviously satisfies the above properties. Then we have:

$$
\mathrm{D}\left(-\omega_{0}\right)^{\vee}=\left\{\sum_{n=i}^{j} \alpha_{n}^{\vee} \mid i \leq 0 \leq j\right\} .
$$

We can identify $\left(\mathrm{D}\left(-\omega_{0}\right)^{\vee} ; \leq\right)$ with a poset $(\mathbb{Y} ; \geq)$, and $\left(\mathrm{D}\left(-\omega_{0}\right)^{\vee} ; \leq\right)$-partitions with plane partitions. Hence, the identity (7.1) can be rewritten as:

$$
T(\mathbb{Y} ; \geq)=T\left(\mathrm{D}\left(-\omega_{0}\right)^{\vee} ; \leq\right)=\prod_{i \leq 0 \leq j} \frac{1}{1-\mathbf{q}^{\sum_{i \leq n \leq j} \alpha_{i}}} .
$$

Similarly, the identity $(7.2)$ can be rewritten as:

$$
U(\mathbb{Y} ; \geq)=U\left(\mathrm{D}\left(-\omega_{0}\right)^{\vee} ; \leq\right)=\prod_{i \leq 0 \leq j} \frac{1}{1-q^{j-i+1}}=\prod_{n \geq 1}\left(\frac{1}{1-q^{n}}\right)^{n} .
$$

The identity (7.4) is known as MacMahon's identity (see [6]) for the generating function of plane partitions.
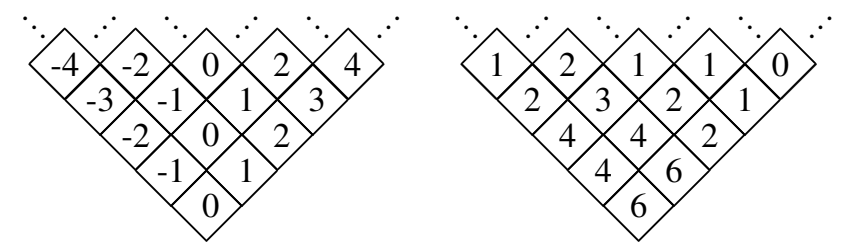

Fig. 7.1: The coloring of $(\mathbb{Y} ; \geq)$ and an example of plane partition

Remark 7.1 We get similar results for infinite Dynkin diagrams $D_{\infty}$ and $B_{\infty}$ below.

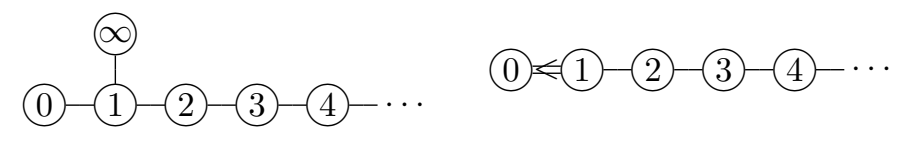

These give the identities for the generating function of shifted plane partitions. For example, a "shifted version" of the identity (7.4) can be written as:

$$
U(\mathbb{S} ; \geq)=\prod_{n \geq 1}\left(\frac{1}{1-q^{n}}\right)^{\lceil n / 2\rceil}=\prod_{n \geq 1}\left(\frac{1}{1-q^{2 n-1}} \frac{1}{1-q^{2 n}}\right)^{n} .
$$



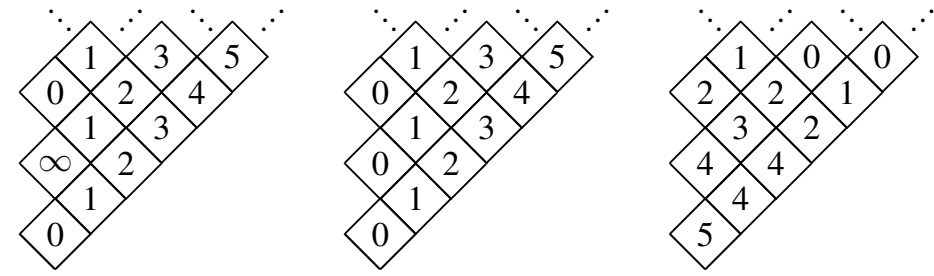

Fig. 7.2: The coloring of type $D$ and of type $B$ of $(\mathbb{S} ; \geq)$, and an example of shifted plane partition

\section{References}

[1] J. B. Carrell, Vector fields, flag varieties and Schubert calculus, Proc. Hyderabad Conference on Algebraic Groups (ed. S.Ramanan), Manoj Prakashan, Madras, 1991.

[2] J. S. Frame, G. de B. Robinson, and R. M. Thrall, The hook graphs of symmetric group, Canad. J. Math. 6 (1954),316-325.

[3] E. R. Gansner, The Hillman-Grassl correspondence and the enumeration of reverse plane partitions, J. Combin. Theory, Ser. A 30 (1981), 71-89.

[4] A. P. Hillman, and R. M. Grassl, Reverse plane partitions and tableau hook numbers, J. Combinatorial Theory A 21 (1976), 216-221.

[5] V. G. Kac, "Infinite Dimentional Lie Algebras,” Cambridge Univ. Press, Cambridge, UK, 1990.

[6] I. G. Macdonald, "Symmetric Functions and Hall Polynomials", Oxford University Press, Oxford, 1999.

[7] R. V. Moody and A. Pianzola, "Lie Algebras With Triangular Decompositions," Canadian Mathematical Society Series of Monograph and Advanced Text, 1995.

[8] K. Nakada, q-Hook formula for a generalized Young diagram, preprint.

[9] K. Nakada, Colored hook formula for a generalized Young diagram, Osaka J. of Math. Vol.45 No.4 (2008), 1085-1120.

[10] R. A. Proctor, Dynkin diagram classification of $\lambda$-minuscule Bruhat lattices and of $d$-complete posets, J.Algebraic Combin. 9 (1999), 61-94.

[11] http://www.math.unc.edu/Faculty/rap/Hook.html

[12] R. P. Stanley, Ordered structures and partitions, Memoirs of the Amer. Math. Soc. No. 119 (1972).

[13] J. R. Stembridge, Minuscule elements of Weyl groups, J.Algebra 235 (2001), 722-743. 